\title{
The anti-IgE antibody omalizumab improves asthma-related quality of life in patients with allergic asthma
}

\author{
R. Buhl*, G. Hanf ${ }^{\#}$, M. Solèr ${ }^{\ddagger}$, G. Bensch ${ }^{+}$, J. Wolfe ${ }^{\S}$, F. Everhard ${ }^{f}$, K. Champain**, H. Fox**, \\ J. Thirlwell**
}

The anti-IgE antibody omalizumab improves asthma-related quality of life in patients with allergic asthma. R. Buhl, G. Hanf, M. Solèr, G. Bensch, J. Wolfe, F. Everhard, K. Champain, H. Fox, J. Thirlwell. (C) ERS Journals Ltd 2002.

ABSTRACT: The aim of the present study was to determine the effect of treatment with omalizumab, an anti-immunoglobulin $E$ antibody, on asthma-related quality of life (AQoL) in patients with moderate-to-severe allergic asthma.

A total of 546 patients with allergic asthma were randomised to double-blind subcutaneous treatment with either placebo or omalizumab for 52 weeks. A constant beclomethasone dipropionate dose was maintained during the first 16 weeks (steroidstable phase). This was followed by a 12-week steroid-reduction phase. The core study was followed by a 24-week double-blind extension phase. AQoL was evaluated at baseline and at the end of the steroid-stable (week 16), steroid-reduction (week 28) and extension phases (week 52) using the Juniper Asthma Quality of Life Questionnaire (AQLQ).

Baseline AQLQ scores were comparable for the two treatment groups. Relative to placebo, omalizumab-treated patients demonstrated statistically significant improvements from baseline across all four AQLQ domains, as well as overall AQoL score, at weeks 16 (except environmental exposure), 28 and 52. Patients on omalizumab were also more likely to achieve clinically significant improvements in AQoL during the course of the study. Overall, almost $70 \%$ of patients and investigators rated treatment with omalizumab as "excellent/good", compared with $\sim \mathbf{4 0} \%$ of placebo recipients.

Clinical studies show that omalizumab enhances disease control whilst reducing corticosteroid consumption in patients with allergic asthma. The results of the present study show that these changes are paralleled by improvements in asthma-related quality of life that are meaningful to such patients.

Eur Respir J 2002; 20: 1088-1094.

\begin{abstract}
*Pulmonary Division, University Hospital, Mainz, and "Allergy and Asthma Clinic, Charité Berlin, Germany. "Pulmonary Division, University Hospital, Basel, Switzerland. 'Allergy, Immunology \& Asthma Medical Group, Stockton, CA, and ${ }^{\$}$ Allergy \& Asthma Associates, San Jose, CA, USA. ${ }^{f}$ Novartis Pharma AG, Basel, Switzerland. **Novartis Horsham Research Centre, Horsham, UK.
\end{abstract}

Correspondence: R. Buhl, Pulmonary Division, University Hospital, Langenbeckstrasse 1, D-55131 Mainz, Germany.

Fax: 496131175545

E-mail: R.Buhl@3-med.klinik.

uni-mainz.de

Keywords: Allergic asthma, antiimmunoglobulin E, omalizumab, quality of life

Received: February 252002

Accepted after revision: June 272002

This study was supported by Novartis Pharma AG, Basel, Switzerland and Genentech Inc., South San Francisco, CA, USA.
It is well established that asthma-related symptoms and the ever-present risk of serious exacerbations requiring emergency treatment and/or hospitalisation exert a profoundly negative effect on various aspects of quality of life (QoL) [1, 2]. Many clinical trials involving patients with asthma have therefore assumed that an improvement in symptoms and other conventional clinical indices (e.g. spirometry) leads to concomitant changes in the patient's QoL. However, whilst providing valuable information about the status of the affected organ system, conventional clinical measures rarely fully capture the extent of the patient's functional impairment and decreased wellbeing $[3,4]$. Thus, in order to obtain a holistic view of the patient's health status, both conventional clinical measures and the patient's health-related QoL should be evaluated during asthma therapy.

The pathophysiology of asthma is allergy related in $>90 \%$ of cases [5]. Central to the mechanisms of allergic inflammation is the release of a number of pro-inflammatory mediators after allergen binding to immunoglobulin $(\mathrm{Ig}) \mathrm{E}$ on the surface of effector cells
[6-10]. Targeting of IgE therefore represents a promising basis for the development of new therapeutic agents for the treatment of allergic asthma. Moreover, stopping the allergic cascade at this early stage may bring about improvements in other IgE-mediated allergic (atopic) diseases, such as seasonal allergic rhinitis, which is highly prevalent in patients with allergic asthma [11].

Omalizumab is the first anti-IgE agent to undergo clinical evaluation in the treatment of IgE-mediated diseases [12]. A recombinant humanised monoclonal anti-IgE antibody [13], omalizumab forms complexes with circulating free $\operatorname{IgE}$ and prevents it binding to high- and low-affinity receptors on effector cells, thereby inhibiting allergen-induced activation [14-16]. Indeed, the pronounced reduction in serum-free IgE concentrations after the first injection of omalizumab in patients with allergic asthma [17] attenuates both the early and late asthmatic responses to inhaled allergen [18, 19].

Recently, the authors reported that subcutaneous treatment with omalizumab improved asthma control 
and allowed a significant reduction in the dose of inhaled corticosteroids in a large placebo-controlled trial in patients with moderate-to-severe allergic asthma $[20,21]$. In the present study, the findings of the effect of omalizumab on asthma-related QoL (AQoL), which was evaluated in parallel with routine clinical indices in this clinical trial, are reported.

\section{Materials and methods}

\section{Study design and patients}

The present QoL study was conducted as part of a multicentre, randomised, double-blind, placebocontrolled clinical trial with omalizumab, for which the protocol and main clinical/tolerability findings are reported in full elsewhere $[20,21]$. Briefly, the study enrolled patients aged 12-75 yrs with a diagnosis of moderate-to-severe allergic asthma of $\geqslant 1 \mathrm{yr}^{\prime}$ s duration, a total serum IgE level of 30-700 International Unit (IU) $\cdot \mathrm{mL}^{-1}$ and a positive skin-prick test to at least one common allergen (Dermatophagoides farinae, D. pteronyssinus, dog or cat). All patients had stable disease and required regular treatment with inhaled corticosteroids (dose equivalent to $500-1,200 \mu \mathrm{g} \cdot \mathrm{day}^{-1}$ of beclomethasone dipropionate (BDP)), but were symptomatic even with such treatment. After screening, patients entered a run-in period of 4-6 weeks, during which time all patients were switched to inhaled BDP and the dose was adjusted to establish the lowest dose required for control of asthma symptoms and peak expiratory flow (PEF) at levels acceptable to the patient and investigator. Patients were maintained on this dose of BDP for the final 4 weeks of run-in (baseline). Eligible patients were subsequently randomised to subcutaneous treatment with either placebo or omalizumab every 2 or 4 weeks $\left(\geqslant 0.016 \mathrm{mg} \cdot \mathrm{kg}^{-1} \cdot \mathrm{IgE}^{-1}\left(\mathrm{IU} \cdot \mathrm{mL}^{-1}\right)\right.$ per 4 weeks $)$ for 52 weeks. The baseline dose of concomitant BDP was maintained during the first 16 weeks of the study (steroid-stable phase). In the following 12 weeks (steroid-reduction phase), the BDP dose was reduced by $25 \%$ every 2 weeks over the first 8 weeks until total elimination, or until there was a decrease in forced expiratory volume in one second (FEV1) of $\geqslant 20 \%$ compared to the last measurement of the previous phase or the development of an event defining asthma worsening, as follows: 1) deterioration of symptoms requiring an urgent or unscheduled physician visit; 2) PEF of $\leqslant 50 \%$ of patient's personal best; 3) decrease in morning PEF of $\geqslant 20 \%$, compared to the last week of the previous phase, on two or more of 3 successive days; 4) increase in rescue medication of $\geqslant 50 \%$, compared to the average use for the last week of the previous phase and exceeding eight puffs of salbutamol per day, on 2 or more of 3 successive days; 5) more than 2 of 3 successive nights disrupted due to asthma symptoms requiring rescue medication.

If there was a decrease in FEV1 of $\geqslant 20 \%$, or asthma worsening occurred, then the previous dose of BDP was resumed. Once the lowest effective dose of BDP for asthma control was established, patients were maintained on this dose for the remaining 4 weeks of the core study. The use of rescue inhaled salbutamol was permitted throughout the core study.

The core study was followed by a 24 -week doubleblind extension during which patients continued on randomised treatment and the lowest effective dose of BDP (which could be adjusted accordingly). During the extension phase, the use of concomitant asthma medication was liberalised and investigators were allowed to administer additional asthma medication and/or switch patients to other asthma medications if deemed necessary.

The study was conducted in accordance with the Declaration of Helsinki. All patients (or their parents or guardians, when appropriate) provided written informed consent prior to the initiation of study. Ethical approval was obtained from the relevant institutional review board of each study centre.

\section{Measurement of asthma-related quality of life}

AQoL was assessed at baseline and at the end of the steroid-stable, steroid-reduction and extension phases using the disease-specific Juniper Asthma Quality of Life Questionnaire (AQLQ) [22]. This questionnaire, which has good reliability and responsiveness with excellent cross-sectional and longitudinal validity [23], comprises 32 questions ("items") grouped into four categories ("domains"): activity limitations (11 items), emotions (five items), symptoms (12 items), and exposure to environmental stimuli (four items). Each question was answered by the patient on a 7-point scale according to the level of impairment in the preceding 2 weeks, from 1 (extremely impaired) to 7 (no impairment); lower AQLQ scores therefore reflect increased impairment. To avoid logistical problems, all patients completed the adult version of the AQLQ (regardless of their age).

\section{Evaluation of treatment effectiveness}

Upon completion of the core study, subjective and objective global evaluations of treatment effectiveness were determined by investigators and patients as "excellent" (complete control of asthma), "good" (marked improvement of asthma), "moderate" (discernible but limited improvement in asthma), "poor" (no appreciable change in asthma) or "worse".

\section{Statistical analysis}

Statistical analyses were performed for all randomised patients. The "last observation carried forward" approach was used within a phase for patients who discontinued prematurely from the study. The main analysis concerned the change from baseline in domain scores and overall score to the end of the steroid-stable, steroid-reduction and extension phases. Between-treatment differences were evaluated using an analysis of covariance model, fitting treatment, centre and dosing schedule as factors and baseline as a covariate. All data from the extension period were analysed post hoc. Further analyses were performed to determine the proportion of patients in each treatment 
group achieving a clinically significant improvement in QoL (i.e. an increase in domain or overall $\mathrm{QoL}$ score of $\geqslant 0.5$ points) as well as those achieving a large improvement (increase in score of $\geqslant 1.5$ points relative to baseline) [24]. This analysis was performed post hoc using a Chi-squared test. The between-treatment analyses of patient and investigator global evaluations of treatment effectiveness at the end of the core study were performed using the generalised CochranMantel-Haenszel (van Elteren) test, stratified by treatment schedule. Statistical significance was accepted when $\mathrm{p}<0.05$ (two-sided).

\section{Results}

\section{Patients}

A total of 546 patients from 42 centres in nine countries completed the run-in/baseline phase and were randomised to study medication: omalizumab, $\mathrm{n}=274$; placebo, $\mathrm{n}=272$. A total of 487 patients $(89 \%)$ completed the core study: omalizumab, $n=255(93 \%)$; placebo, $\mathrm{n}=232(85 \%)$. Most patient withdrawals in each treatment group occurred during the initial 16-week steroid-stable phase: omalizumab, $n=13$; placebo, $n=27$. Withdrawal of consent was the most common reason for discontinuation.

A total of 483 patients continued into the extension phase of the study: omalizumab, $n=254$; placebo, $\mathrm{n}=229$. Nearly three times as many patients in the placebo group $(\mathrm{n}=26(11.4 \%))$ as in the omalizumab group $(\mathrm{n}=10(3.9 \%))$ discontinued prematurely during the extension. Withdrawal of consent and loss to follow-up were the most frequent reasons for discontinuation, affecting more placebo than omalizumab patients in both cases.

Overall, the two treatment groups were well balanced in terms of demographics and baseline clinical characteristics, including mean AQLQ domain and overall scores (table 1). Both treatment groups included a comparable proportion of minor protocol violators, the inclusion of which was not deemed to have affected the results of the study.

\section{Asthma-related quality of life}

Relative to placebo, mean AQLQ domain and overall scores showed a progressive increase throughout 52-weeks' treatment with omalizumab, consistent with an improvement in AQoL (fig. 1). The principal analysis, however, concerned the change from baseline in such scores during omalizumab therapy relative to placebo. Overall, the change from baseline in AQLQ domain scores for activities, emotions and symptoms, as well as overall score, showed significant improvement over placebo during the 16 weeks of the steroidstable phase (fig. 2). Improvements in AQoL with omalizumab were sustained during the 12 weeks of the steroid-reduction phase and showed a progressive improvement across all four domains and with regard to the overall score (fig. 2). Improvements in AQoL with omalizumab were also sustained during the 24week extension phase (fig. 2).

Compared with placebo, a greater proportion of patients on omalizumab achieved a clinically significant improvement in AQoL during each phase of the study (fig. 3). The proportion of patients achieving a "large" improvement in AQoL, defined as an increase in domain or overall QoL score of $\geqslant 1.5$ points [24], is shown in figure 3. In terms of overall score, the proportion of patients who achieved a large improvement in AQoL was significantly higher than placebo during each phase of the study (fig. 3).

\section{Treatment effectiveness}

The improvement in asthma control [20, 21] and AQoL with omalizumab therapy was paralleled by patient and investigator opinions of treatment effectiveness at the end of the core study, which showed significant superiority for omalizumab relative to placebo (both $\mathrm{p}<0.001$ ). Almost $70 \%$ of patients and investigators rated treatment with omalizumab as

Table 1. - Demographics and baseline clinical characteristics (intent-to-treat population)

\begin{tabular}{|c|c|c|}
\hline & Omalizumab & Placebo \\
\hline Sex $\%$ male & 51 & 47 \\
\hline Mean age yrs & $40(12-76)$ & $39(12-72)$ \\
\hline Mean duration of asthma yrs & $20.3(2-68)$ & $19.1(1-63)$ \\
\hline Mean BDP dose $\mu \mathrm{g} \cdot \mathrm{day}^{-1}$ & $769(500-1600)$ & $772(200-2000)$ \\
\hline Mean serum total IgE IU $\cdot \mathrm{mL}^{-1}$ & $223(21-785)$ & $206(22-814)$ \\
\hline Mean FEV1 \% pred & $70(30-112)$ & $70(22-109)$ \\
\hline Severe asthma n $(\%)^{\#}$ & $60(22)$ & $59(22)$ \\
\hline \multicolumn{3}{|l|}{ Mean AQLQ domain scores } \\
\hline Activities & $4.41(1.27-7.00)$ & $4.36(1.67-6.82)$ \\
\hline Emotions $_{s}^{+}$ & $4.76(1.40-7.00)$ & $4.65(1.00-7.00)$ \\
\hline Symptoms ${ }^{\S}$ & $4.47(1.45-6.70)$ & $4.39(2.00-6.42)$ \\
\hline Environmental exposure ${ }^{f}$ & $4.48(1.00-7.00)$ & $4.25(1.00-7.00)$ \\
\hline Overall score & $4.43(1.58-6.48)$ & $4.36(1.84-6.38)$ \\
\hline
\end{tabular}

Data are presented with range in parentheses unless otherwise stated. Omalizumab n=274, placebo n=272. BDP: beclomethasone dipropionate; IgE: immunoglobulin E; IU: International Units; FEV1: forced expiratory volume in one second; \% pred: \% predicted; AQLQ: Asthma Quality of Life Questionnaire. ${ }^{\#}$ : baseline FEV1 $\leqslant 65 \%$ pred and a mean total symptom score of $>4$ for the last 2 weeks of the run-in period; 9 : omalizumab $n=258$, placebo $n=251 ;{ }^{+}:$omalizumab $n=221$, placebo $n=212 ;$; : omalizumab $\mathrm{n}=222$, placebo $\mathrm{n}=212{ }^{f}$ : omalizumab $\mathrm{n}=222$, placebo $\mathrm{n}=211$; ${ }^{\# \#}$ : omalizumab $\mathrm{n}=257$, placebo $\mathrm{n}=251$. 

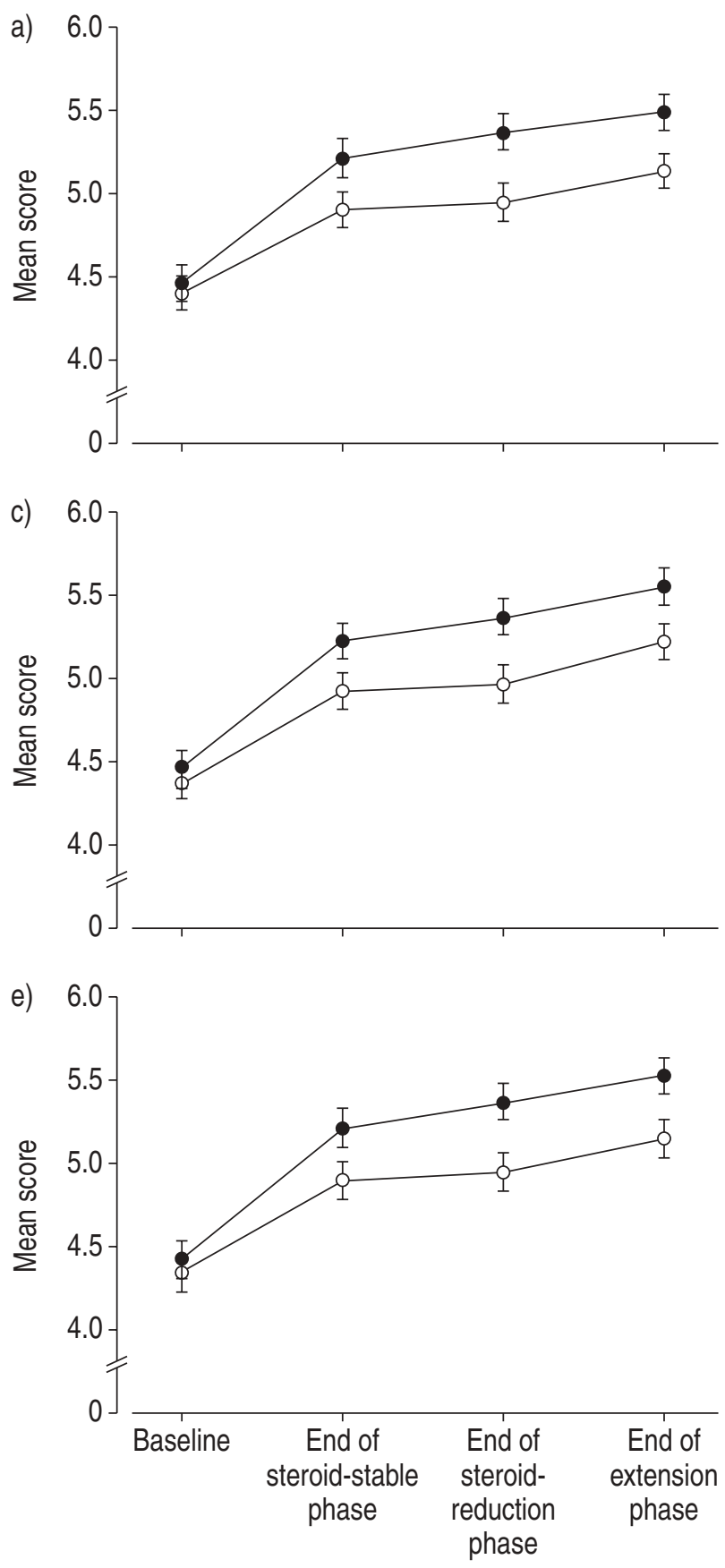

"excellent/good", compared with $\sim 40 \%$ of those on placebo (fig. 4). These effects were obtained even though there was a significantly greater reduction in the dose of inhaled corticosteroid in the omalizumabtreated patients.

\section{Discussion}

It is becoming increasingly recognised amongst healthcare providers that asthma causes significant impairment of physical and psychosocial well-being in the affected individual. Consequently, improving the health-related QoL of patients should be one of the

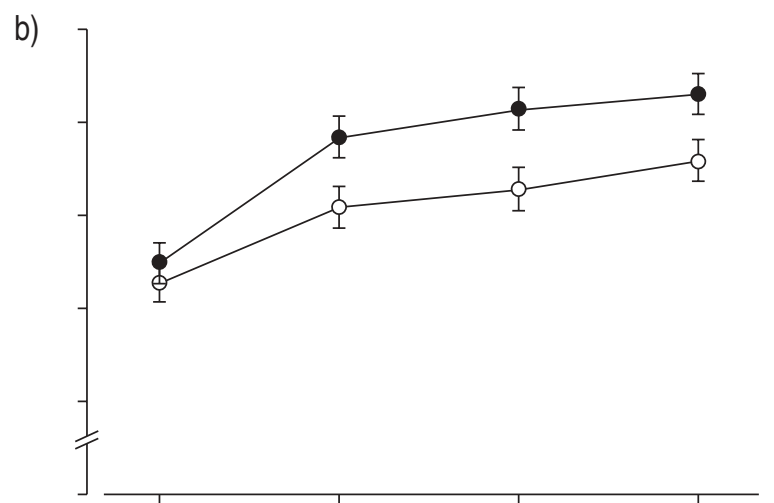

d)

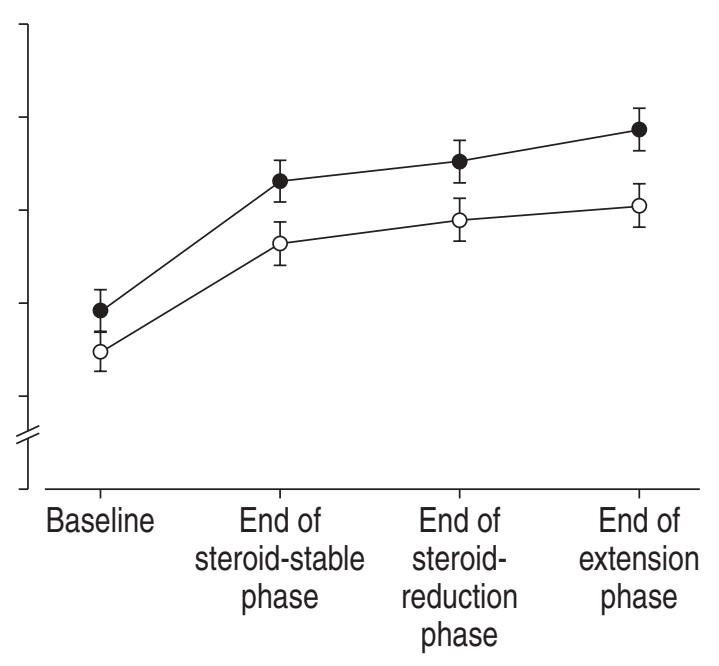

Fig. 1.-Mean domain and overall asthma-related quality-oflife scores for a) activities, b) emotions, c) symptoms, d) exposure and e) overall, as determined by the Juniper Asthma Quality of Life Questionnaire, during treatment with omalizumab (O) or placebo $(\bigcirc)$ in patients with moderate-to-severe allergic asthma.

primary goals in asthma treatment. This is particularly important because traditional clinical parameters, such as lung function, have, at best, only weak associations with QoL $[4,25]$, an understanding that has led to a more holistic approach to management in recent years. Assessment of health-related QoL, in addition to standard clinical indices of airways status, has therefore become an integral part of the determination of treatment response in asthma patients. Omalizumab, a recombinant humanised monoclonal anti- $\operatorname{IgE}$ antibody, is the first anti-IgE agent to undergo clinical evaluation in the treatment of allergic asthma. The current authors have previously reported that the addition of this agent to the therapeutic regimen 

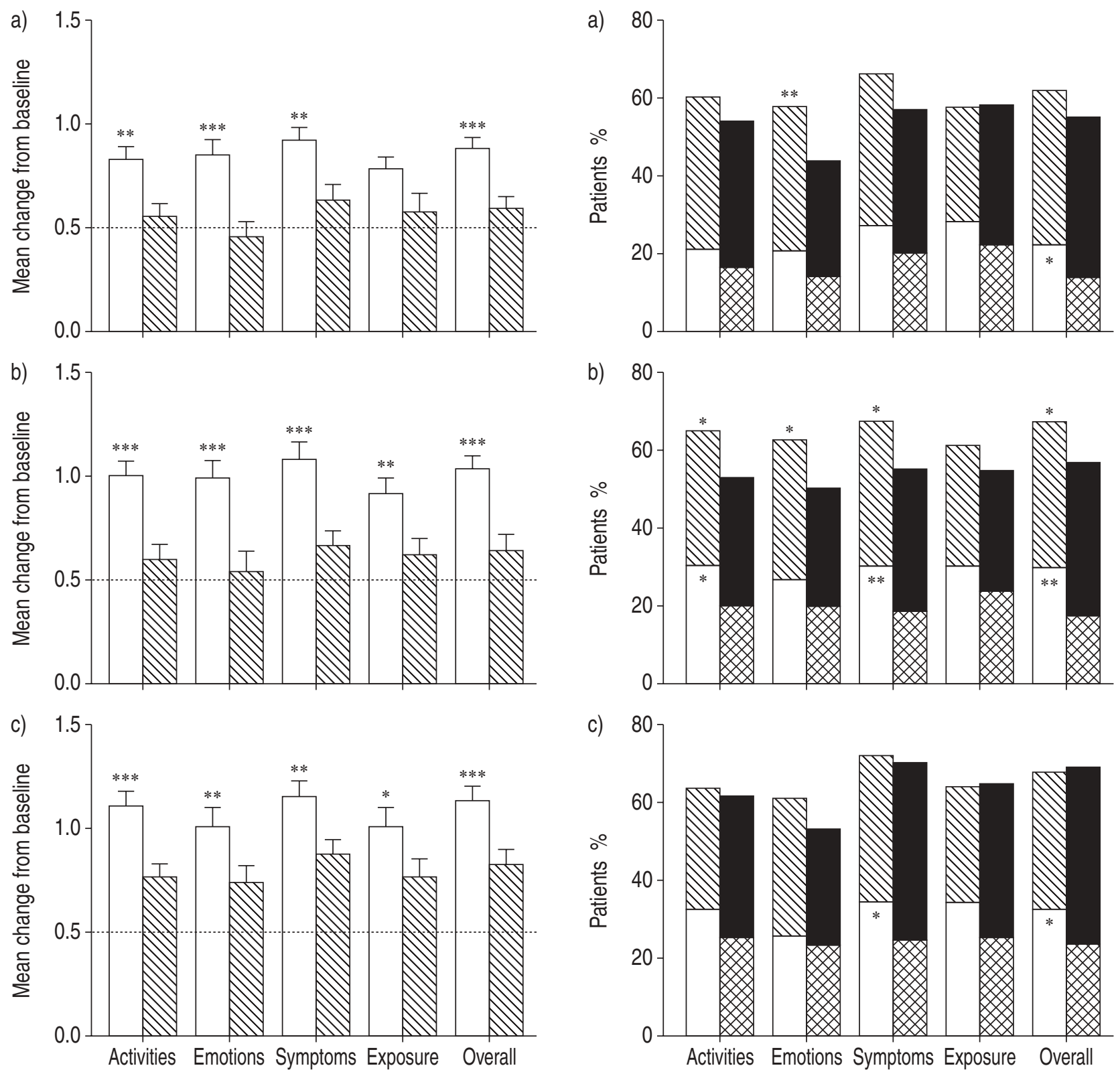

Fig. 2.-Adjusted mean change from baseline in asthma-related quality-of-life scores, as determined by the Juniper Asthma Quality of Life Questionnaire, during treatment with omalizumab $(\square)$ or placebo $(\mathbb{\mathbb { S }})$ in patients with moderate-to-severe allergic asthma. a) End of steroid-stable phase, b) end of steroid-reduction phase and c) end of extension phase. An increase in score of $\geqslant 0.5$ points from baseline indicates a clinically significant improvement $(\cdots$.$) [24]. *: \mathrm{p}<0.05$ versus placebo; $* *: \mathrm{p}<0.01$ versus placebo; $* * *: \mathrm{p}<0.001$ versus placebo.

of patients with allergic asthma simultaneously reduces both asthma exacerbations and inhaled corticosteroid requirement, whilst improving other parameters of disease control [20,21]. The findings of the present QoL study, performed in parallel with the clinical evaluations in this clinical trial, show that such changes are clinically significant to patients, with marked improvement in all aspects of their AQoL.

The authors utilised the AQLQ in the present study, a rigorous questionnaire that was specifically designed

Fig. 3. - Percentage of patients achieving minimal ${ }^{\#}$ or large clinically significant improvement in quality-of-life scores during treatment with omalizumab or placebo in patients with moderate-to-severe allergic asthma. a) End of steroid-stable phase, b) end of steroidreduction phase and c) end of extension phase. $\mathbb{Q}$ and $\square: \geqslant 0.5$; $\square: \geqslant 1.5$ with omalizumab; $\mathbf{\square}$ and $\geqslant 0.5 ; \mathbf{\square}: \geqslant 1.5$ with placebo. \#: defined as an increase in score of $\geqslant 0.5$ points from baseline [24]; : defined as an increase in score of $\geqslant 1.5$ points from baseline [24]. *: $\mathrm{p}<0.05$ versus placebo; ${ }^{* *}: \mathrm{p}<0.01$ versus placebo.

to be sensitive to small, within-subject changes in AQoL over time. With this questionnaire, an increase in domain or overall score of $\geqslant 0.5$ points indicates a clinically significant improvement in AQoL, with increases from baseline of $\geqslant 1.5$ points reflecting a "large" improvement [24]. This analytical approach allows a meaningful interpretation of the effect of therapeutic intervention on AQoL. Repeated use of questionnaires is as valid for measuring asthma control as daily diary data [26]. In the present study, 

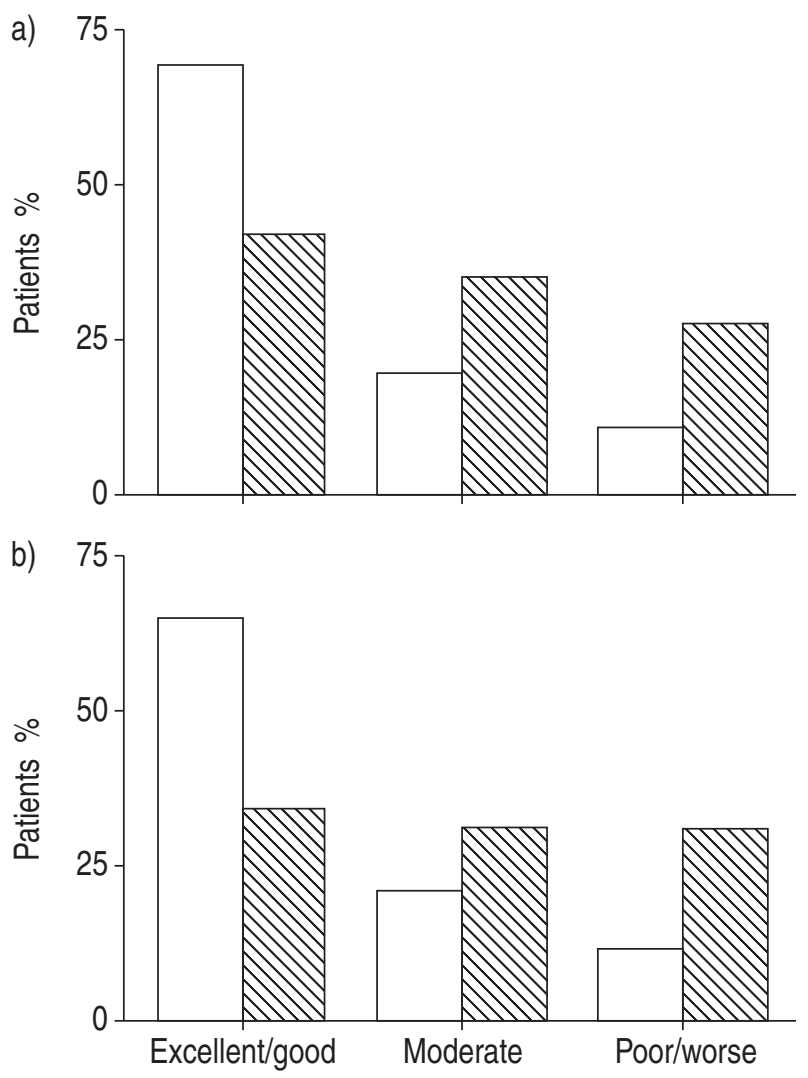

Fig. 4.-a) Patient and b) investigator global evaluations of treatment effectiveness after 28 weeks' treatment with omalizumab $(\square)$ or placebo $(\mathbb{\nabla})$ in patients with moderate-to-severe allergic asthma. $\mathrm{p}<0.001$ for both.

it is evident that, compared with placebo, a greater proportion of patients on omalizumab achieved a clinically significant improvement in AQoL during each phase of the study. Moreover, with regard to overall AQoL score, the proportion of patients who achieved a large improvement in AQoL was significantly greater in patients on omalizumab than on placebo during each phase of the study. It is worth noting that the clinically significant improvement in QoL with omalizumab was achieved despite the significant concomitant reduction in reliance on inhaled corticosteroids $[20,21]$. Such findings provide compelling evidence that omalizumab targets a cause of the underlying allergic disease, thereby permitting enhanced asthma control, which consequently leads to a clinically significant improvement in AQoL. This has considerable clinical importance given the potential side-effects of long-term therapy with higher doses of inhaled corticosteroids, which are a real concern in the treatment of this chronic disease [27]. The fact that omalizumab therapy was highly rated by patients and investigators alike reinforces these findings.

As observed for the clinical indices of treatment efficacy $[20,21]$, notable improvements in AQoL occurred among some patients on placebo. Such findings have been observed in previous placebocontrolled AQoL studies in asthma patients [28], although a marked placebo-effect response is not unique to the treatment of this disease [29]. One possible explanation for the high placebo response in the present study is improved compliance with BDP treatment. Unfortunately, it is impossible to avoid the improved compliance trap unless clinical trials are preceded by a very long (months) run-in period during which compliance is carefully monitored and a significant percentage of patients can be lost due to the very improvements seen in the present study. Indeed, close monitoring by the investigator, with repeated encouragement to adhere to therapeutic regimens combined with perfect and immediate treatment of any complaint of the patient (be it related to asthma or not), undoubtedly contributed towards the significant placebo response. Against this background, adding omalizumab was still able to significantly improve both disease control and AQoL. The optimal utility of this agent may therefore be apparent in patients who are poorly controlled despite optimal therapy and good compliance.

During the extension phase it was apparent that, while the improvement in AQLQ domain and overall scores versus baseline was still significantly higher for those receiving active treatment (see fig. 2), the differences to placebo were less marked compared to the other two phases. This result was not entirely unexpected, as patients were permitted to use concomitant asthma medication throughout the extension phase, and the placebo-treated patients used more of such medications than those randomised to omalizumab [21]. For example, some $85 \%$ of placebo recipients received inhaled corticosteroids during the extension phase, while $17 \%$ and $3.5 \%$ of patients, respectively, were treated with additional long-acting $\beta_{2}$-agonists and leukotriene receptor antagonists. Corresponding values for the omalizumab treatment arm were $65 \%, 11 \%$ and $0.4 \%$, respectively [21]. Another factor likely to have contributed to the increased placebo response during the extension phase was the higher discontinuation rate among placebo recipients, which may have served to increase the proportion of patients with a good placebo response. Moreover, the additional benefit of omalizumab becomes clearer when looking at the proportion of patients who achieved a large, clinically relevant improvement in AQoL and the patient and investigator opinions of treatment effectiveness, both of which showed significant superiority for omalizumab than placebo.

The present study enrolled adolescent ( $>12$ yrs old) and adult patients with allergic asthma. In order to avoid the methodological problem of using two different questionnaires in the same trial, the adult version of the AQLQ was used exclusively in the present trial. Only 35 patients aged 12-17 yrs were enrolled and were evenly distributed between the two groups. An analysis including and excluding these subjects showed that they did not influence the results of the present study.

As previously mentioned, improved patient compliance with prescribed medication may partly explain an improvement in disease management and hence AQoL. Indeed, existing treatment with inhaled corticosteroids and bronchodilators may be complicated by the use of multiple inhalers with different administration 
schedules that can give rise to poor patient compliance. With convenient subcutaneous administration every 2 or 4 weeks by their attending physician, treatment with omalizumab may overcome the potential for poor compliance with anti-asthma therapy.

Whilst the main tolerability findings of this study are reported in full elsewhere [20,21], it is important to re-consider the side-effects of omalizumab in relation to any possible impact they may have on QoL. Overall, the side-effects of omalizumab and placebo were similar with no clinically relevant differences. As such, the tolerability profile of omalizumab is unlikely to have had a negative effect on the QoL findings of the present study.

In conclusion, the results of the present study show that omalizumab therapy is associated with clinically significant improvement in all aspects of asthmarelated quality of life in patients with moderate-tosevere allergic asthma. The improvement in patient functioning and well-being paralleled earlier findings of enhanced disease control, even though there was a significant reduction in inhaled corticosteroid consumption, during omalizumab therapy in this patient population $[20,21]$. Taken together, these findings indicate that omalizumab is a promising new agent for the treatment of allergic asthma.

\section{References}

1. Juniper EF. Quality of life in adults and children with asthma and rhinitis. Allergy 1997; 52: 971-977.

2. Juniper EF. Effect of asthma on quality of life. Can Respir J 1998; 5: Suppl. A, 77A-84A.

3. Juniper EF, Johnston PR, Borkhoff CM, Guyatt GH, Boulet LP, Haukioja A. Quality of life in asthma clinical trials: comparison of salmeterol and salbutamol. Am J Respir Crit Care Med 1995; 151: 66-70.

4. Moy ML, Israel E, Weiss ST, Juniper E, Dube L, Drazen JM and and the NHLBI Asthma Clinical Research Network. Clinical predictors of healthrelated quality of life depend on asthma severity. Am J Respir Crit Care Med 2001; 163: 924-929.

5. Holt PG, Macaubas C, Stumbles PA, Sly PD. The role of allergy in the development of asthma. Nature 1999; 402: Suppl., B12-B17.

6. Drazen JM, Arm JP, Austen KF. Sorting out the cytokines of asthma. Commentary. J Exp Med 1996; 183: $1-5$.

7. Hamelmann E, Tadeda K, Oshiba A, Gelfand EW. Role of $\operatorname{IgE}$ in the development of allergic airway inflammation and airway hyperresponsiveness - a murine model. Allergy 1999; 54: 297-305.

8. Spector SL. Allergic inflammation in upper and lower airways. Ann Allergy Asthma Immunol 1999; 83: 435-444.

9. Wills-Karp M. Immunologic basis of antigen-induced airway hyperresponsiveness. Ann Rev Immunol 1999; 17: 255-281.

10. Williams CM, Galli SJ. The diverse potential effector and immunoregulatory roles of mast cells in allergic disease. J Allergy Clin Immunol 2000; 105: 847-859.

11. The International Study of Asthma and Allergies in Childhood (ISAAC) Steering Committee. Worldwide variation in prevalence of symptoms of asthma, allergic rhinoconjunctivitis, and atopic eczema: ISAAC. Lancet 1998; 351: 1225-1232.
12. Easthope S, Jarvis B. Omalizumab. Drugs 2001; 61: 253-260.

13. Presta LG, Lahr SJ, Shields RL, et al. Humanization of an antibody directed against IgE. J Immunol 1993; 151: 2623-2632.

14. Shields RL, Werther WR, Zioncheck K, et al. AntiIgE monoclonal antibodies that inhibit allergenspecific histamine release. Int Arch Allergy Immunol 1995; 107: 412-413.

15. Coyle AJ, Wagner K, Bertrand C, Tsuyuki S, Bews J, Heusser C. Central role of immunoglobulin (Ig) $\mathrm{E}$ in the induction of lung eosinophil infiltration and $\mathrm{T}$ helper 2 cell cytokine production: inhibition by a nonanaphylactogenic anti-IgE antibody. J Exp Med 1996; 183: $1303-1310$

16. Jardieu PM, Fick RB Jr. IgE inhibition as a therapy for allergic disease. Int Arch Allergy Immunol 1999; 118: 112-115.

17. Milgrom $\mathrm{H}$, Fick RB, Su JQ, et al. Treatment of allergic asthma with monoclonal anti-IgE antibody. N Engl J Med 1999; 341: 1966-1973.

18. Boulet LP, Chapman KR, Cote J, et al. Inhibitory effects of an anti-IgE antibody E25 on allergeninduced early asthmatic response. Am J Respir Crit Care Med 1997; 155: 1835-1840.

19. Fahy JV, Fleming HE, Wong HH, et al. The effect of an anti-IgE monoclonal antibody on the early- and late-phase responses to allergen inhalation in asthmatic subjects. Am J Respir Crit Care Med 1997; 155: $1828-1834$.

20. Solèr M, Matz J, Townley $\mathrm{R}$, et al. The anti-IgE antibody omalizumab reduces exacerbations and steroid requirement in allergic asthmatics. Eur Respir $J$ 2001; 18: 254-261.

21. Buhl R, Solèr M, Matz $\mathrm{J}$, et al. Omalizumab provides long-term control in patients with moderate-to-severe allergic asthma. Eur Respir J 2002; 20: 73-78.

22. Juniper EF, Guyatt GH, Epstein RS, Ferrie PJ, Jaeschke R, Hiller TK. Evaluation of impairment of health-related quality of life in asthma: development of a questionnaire for use in clinical trials. Thorax 1992; 47: 76-83.

23. Juniper EF, Guyatt GH, Ferrie PJ, Griffith LE. Measuring quality of life in asthma. Am Rev Respir Dis 1993; 147: 832-838.

24. Juniper EF, Guyatt GH, Willan A, Griffith LE. Determining a minimal important change in the disease-specific quality of life questionnaire. J Clin Epidemiol 1994; 47: 81-87.

25. Wijnhoven HA, Kriegsman DM, Hesselink AE, Penninx BW, de Haan M. Determinants of different dimensions of disease severity in asthma and COPD: pulmonary function and health-related quality of life. Chest 2001; 119: 1034-1042.

26. Juniper EF, O'Byrne PM, Ferrie PJ, King DR, Roberts JN. Measuring asthma control. Clinic questionnaire or daily diary? Am J Respir Crit Care Med 2000; 162: 1330-1334.

27. Israel E, Banerjee TR, Fitzmaurice GM, Kotlov TV, LaHive K, LeBoff MS. Effects of inhaled glucocorticoids on bone density in premenopausal women. $N$ Engl J Med 2001; 345: 941-947.

28. Joyce DP, Jackevicius C, Chapman KR, McIvor RA, Kesten S. The placebo effect in asthma drug therapy trials: a meta-analysis. J Asthma 2000; 37: 303-318.

29. Thompson WG. Placebos: a review of the placebo response. Am J Gastroenterol 2000; 95: 1637-1643. 